\title{
QUALITATIVE BEHAVIOUR OF A PHYTOPLANKTON GROWTH MODEL IN A PHOTOBIOREACTOR
}

\author{
Lionel Pawlowski, Olivier Bernard, Emilie Le Floc'h, \\ Antoine Sciandra
}

\author{
COMORE: \\ LOV, BP 28, 06234 Villefranche-sur-Mer, France \\ E \\ INRIA, BP 93, 06902 Sophia-Antipolis Cedex, France \\ fax: +33492387858; e-mail:obernard@sophia.inria.fr
}

\begin{abstract}
A nonlinear light- and nitrate-limited phytoplankton growth model in a photobioreactor is presented. Its qualitative steady input-output behaviour is studied and compared to biological observations of the evolution of ratios between nitrogen, carbon, chlorophyll with respect to the two considered inputs (light and dilution rate). A qualitative validation of the model is provided. The steady input-output behaviour is also used to get linear regressions that provide an estimate of the parameter values. The simulations of the model identified and validated with this approach are consistent with the data.
\end{abstract}

Keywords: photobioreactor, phytoplankton, qualitative analysis, identification, validation.

\section{INTRODUCTION}

The estimation of the amount of $\mathrm{CO}_{2}$ fixed by oceanic phytoplankton during primary production is a key point to quantify the future evolution of atmospheric carbon. Modelling of phytoplankton growth is therefore an important challenge to improve the predictions on planet global warming. Phytoplankton growth is highly dependant on many environmental factors. Light, nutrients and temperature are generally considered as the main factors affecting phytoplankton growth (Chalup and Laws, 1990). In stratified waters, light is available in the surface layer but nitrates are generally present at low concentrations. On the contrary, in the deep layer, light is low and nitrate abundant. For intermediate situations, light and nitrates are both at suboptimal levels for phytoplankton growth. Moreover, due to vertical mixing, cells are continuously exposed to a variable environment, and must adapt their metabolism to sustain an optimal growth under opposing gradients of light and nitrogen. This paper proposes a model that will describe the phytoplanktonic behaviour when both nitrogen and light are at suboptimal level.

Modelling, and therefore validation of biological systems are known to be very delicate. Contrary to other domains (physics, electronics, etc), biological models are not based on admitted laws (like e.g. the Ohm's law in electronics). To circumvent this difficulty, Bastin and Dochain (1990) have introduced mass balance modelling in bioprocesses. It results in splitting the model in two parts: modelling of mass transfers and modelling of biological kinetics. We propose here a method to test the structural validity of our model at steady state. More precisely, we assume that the input-output behaviour of the processes at steady state is qualitatively known and we check whether the model has the same properties. Thus, we must study the response of the equilibria reached by 
the model to changes in the inputs (here light or nitrogen limitation rate). If the model does not fit the qualitative experimental observations, it will be rejected. The method consists of selecting from previous experiments or literature a set of experimental observations describing qualitatively the evolution of the steady states when an input is increased or decreased. In a second step, the model equilibria are computed and their variations and those of nonlinear combinations of state variables are studied with respect to the inputs.

The paper is organised as follows. In a first part, we present the experimental photobioreactor and the mechanism of carbon incorporation. Some experimental facts that should be reproduced by the model are listed. Secondly, we present the main hypotheses of the mass balance model, and we explain how the reaction rates have been chosen. In a third part, we study the steady states and their response to changes of the inputs. Finally we explain briefly the identification process and we present simulations to show that the model agreement with data is also quantitative.

\section{SYSTEM OVERVIEW}

\subsection{Presentation of the photobioreactors}

Photobioreactors are commonly used as culture systems for microalgae with various applications (health food, aquaculture, fine chemicals, environmental applications, etc...). In these systems, the growth rate of the microalgae is controlled by adjusting the environmental conditions of the culture such as light, temperature, nutrient supply... The photobioreactors used for this study consist of 5 liters thermostated vessels fed with sea water whose nitrate $\left(\mathrm{NO}_{3}\right)$ is the limiting substrate. The light intensity is adjusted by filters. The measurements of residual substrate is performed by an automaton related to an autoanalyser Technicon. The cell density is acquired with an automated laser HIAC particle counter. Chlorophyll $a$ (Chl) is estimated with a spectrophotometer (Sciandra et al., 2000) and the concentration of particulate carbon $(C)$ and nitrogen $(N)$ are obtained by a $\mathrm{CHN}$ analyser. For more details on the experimental device, see (Bernard et al., 1996). Data were obtained from experiments of simultaneous limitation by light and nitrate with Rhodomonas salina (Cryptophyceae).

\subsection{The nonlinear coupling between light and nitrogen limitation}

Experimental studies (Rhee and Gotham, 1981) have highlighted the interaction between light and nutrients on growth through a physiological adaptation known as the compensation phenomenon. The cell can compensate a decrease of light intensity by optimising its ability to harvest photons through an increase of its pigment content (Falkowski and Owens, 1980) and by regulating the Ribulose-1,5-bisphosphate carboxylase/oxygenase (Rubisco). Rubisco and chlorophyll represent large nitrogen reserves in the cell and vary with the nitrogen status and light limitation (Sciandra et al., 1997).

\subsection{The experimental steady input-output behaviour}

The following biological features have been observed experimentally at steady state:

- Carbon concentration increases with light and decreases with dilution rate (see Fig. 1a and also see Geider et al.,1998).

- Chlorophyll concentration is a decreasing function of the light due to photoadaptation (Laws and Bannister, 1980). It increases with the dilution rate (see Fig. 1b).

- Chl: $C$ increases with the dilution rate and decreases with light intensity (Chalup and Laws, 1990; Geider et al., 1998)

- $N: C$ increases with the dilution rate (Chalup and Laws, 1990) and decreases with light.

\section{MODEL SET-UP}

\subsection{Introduction}

Many phytoplankton growth models have been developed for one limiting factor such as nutrient, light or temperature. Models integrating more than one limitation are often difficult to validate for two reasons. First, they often use numerous parameters that are difficult to identify from the available data (e.g. (Geider et al., 1998)). Secondly, as they tend to describe detailed cellular processes, state variables often represent quantities that can not be measured experimentally (e.g. (Shuter, 1979)).

Our model integrates both limitations by light and nitrogen. It has been designed to have a simple formulation with few parameters. Three of the four state variables represent measurable quantities in a photobioreactor or in the open sea (chlorophyll, carbon, nitrate, particulate nitrogen). These features permit easier identification and validation.

Phytoplankton growth is assumed to be triggered by two distinct metabolic pathways: uptake and assimilation of nitrogen on one hand and carbon fixation through photosynthesis on the other hand. 

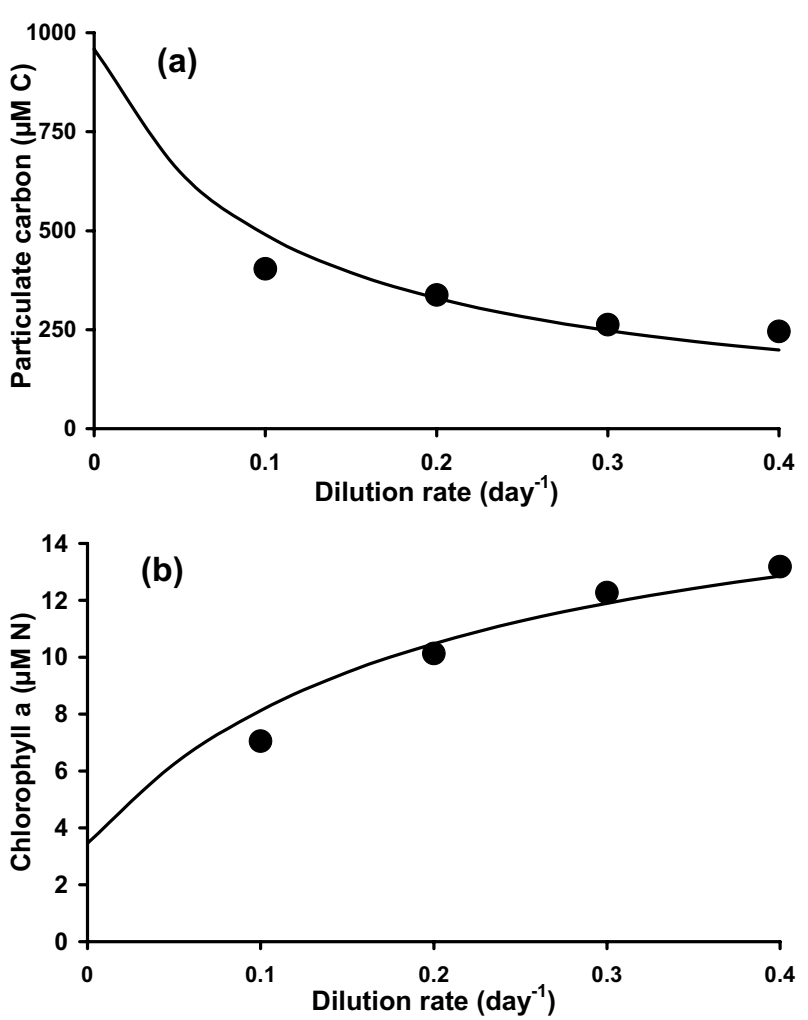

Fig. 1. Steady-state concentrations for particulate carbon (a) and chlorophyll $a$ (b) at different dilution rates for experimental data (points) and calculated equilibria for model state variables (line) (see section 4.1 for details). Steady-state solutions evolve accordingly to observations.

The associated reaction scheme (see (Bastin and Dochain, 1990) for more details on mass balance modelling) for the nitrogen pathway is therefore:

- First, nitrogen source (nitrate, denoted $S$ ) is uptaken by the cell into a cellular nitrogen pool $(N)$ :

$$
S \stackrel{r_{1}(\cdot)}{\longrightarrow} N
$$

The rate of this reaction is denoted $r_{1}($.$) .$

- In a second step, the nitrogen pool is used to produce the chlorophyllian proteins $(L)$ associated with the photosynthesis process:

$$
N \stackrel{r_{2}(.)}{\longrightarrow} L
$$

The rate of this reaction is denoted $r_{2}($.$) .$

- Finally, a natural degradation of the Chlorophyll is also taken into account. The rate of the reaction is $r_{3}($.$) :$

$$
L \stackrel{r_{3}(\cdot)}{\longrightarrow} N
$$

We assume that the carbon pathway results mainly from two reactions:

- Inorganic carbon $\left(\mathrm{CO}_{2}\right)$ is incorporated into the cell through photosynthesis to form particulate carbon $(C)$.

$$
\mathrm{CO}_{2} \stackrel{r_{4}(\cdot)}{\longrightarrow} \mathrm{C}+\mathrm{O}_{2}
$$

The photosynthesis rate is $r_{4}($.

- A proportion of the carbon is lost by respiration:

$$
\mathrm{C}+\mathrm{O}_{2} \stackrel{\mathrm{r}_{5}(\cdot)}{\longrightarrow} \mathrm{CO}_{2}
$$

The model has therefore the general form exposed in Bastin and Dochain (1990):

$$
\frac{d \xi}{d t}=K r(\xi)-D \xi+D \xi_{i n}-Q(\xi)
$$

with the following definitions:

$$
\begin{gathered}
\xi=\left(\begin{array}{c}
S \\
N \\
L \\
C \\
C O_{2} \\
O_{2}
\end{array}\right), \xi_{\text {in }}=\left(\begin{array}{c}
S_{i n} \\
0 \\
0 \\
0 \\
C O_{2 i n} \\
O_{2 i n}
\end{array}\right), r(\xi)=\left(\begin{array}{c}
r_{1}(\xi) \\
r_{2}(\xi) \\
r_{3}(\xi) \\
r_{4}(\xi) \\
r_{5}(\xi)
\end{array}\right), \\
K=\left(\begin{array}{ccccc}
-1 & 0 & 0 & 0 & 0 \\
1 & -1 & 1 & 0 & 0 \\
0 & 1 & -1 & 0 & 0 \\
0 & 0 & 0 & 1 & -1 \\
0 & 0 & 0 & -1 & 1 \\
0 & 0 & 0 & 1 & -1
\end{array}\right), Q(\xi)=\left(\begin{array}{c}
0 \\
0 \\
0 \\
0 \\
Q_{C O_{2}} \\
Q_{O_{2}}
\end{array}\right)
\end{gathered}
$$

Here $D$ is the dilution rate of the bioreactor, $S_{i n}$, $\mathrm{CO}_{2 i n}$ and $\mathrm{O}_{2 i n}$ are respectively the concentration of $\mathrm{S}, \mathrm{CO}_{2}$ and $\mathrm{O}_{2}$ in the renewal medium. The gaseous flow rate of carbon dioxide and oxygen outside the bioreactor are denoted $Q_{\mathrm{CO}_{2}}$ (it is generally negative because of the photosynthesis) and $Q_{O_{2}}$.

\subsection{Kinetic description}

Now we will use the available biological knowledge to estimate the reaction rates with respect to the state variables and to the light intensity $I$ :

$r_{1}$ : The nitrate uptake rate is classically represented by a Monod kinetics (Dugdale, 1967): $r_{1}(\xi)=\rho_{m} \frac{S}{S(t)+K_{S}} C$. Parameters $\rho_{m}$ and $K_{s}$ represent respectively the maximum uptake rate and the half-saturation constant for the absorption of nitrate.

$r_{2}$ : The chlorophyll synthesis is assumed to be dependent on light intensity and on the ratio $\frac{L}{C}$. It is proportional to the nitrogen pool $N: r_{2}(\xi, I)=\gamma(I) N \frac{L}{C}$. We assume that the pigment synthesis is of Monod type at low irradiance and that it is inhibited at high light levels. We take the following expression: $\gamma(I)=\frac{\alpha K_{L} I}{K_{I}+I} \frac{K_{C}}{K_{C}+I}$ where $\alpha$ and $K_{I}$ are respectively the maximum rate for carbon and the half-saturation coefficient for the fixation rate. $K_{L}$ is the maximum synthesis rate and $K_{C}$ is a threshold coefficient. 
$r_{3}$ : The chlorophyll degradation rate is assumed to be constant (with a coefficient $\beta$ ): $r_{3}(\xi)=$ $\beta L$.

$r_{4}$ : The photosynthesis rate is highly dependant on light level and chlorophyll pigments which act as catalyzers for this reaction. Inorganic carbon is considered as non-limiting for phytoplankton growth in marine environment, and therefore it does not intervene:

$r_{4}(\xi, I)=a(I) L$ with $a(I)=\frac{\alpha \cdot I}{K_{I}+I}$. The same coefficients $\alpha$ and $K_{I}$ as these for $\gamma(I)$ have been selected in order to reduce the number of parameters (actually the identified values of these parameters for $\gamma(I)$ and $a(I)$ were quite close). In the sequel, we will consider the quantity $k(I)=\gamma(I) / a(I)$.

$r_{5}$ : The respiration rate is simply proportional to the carbon biomass (with a factor $\lambda$ ): $r_{5}(\xi)=\lambda C$.

From the preceding hypotheses, it follows that inorganic carbon and oxygen do not affect the other state variables. We will therefore consider the following submodel:

$$
\left(\Sigma_{D}\right)\left\{\begin{aligned}
\dot{S}(t)= & D\left[S_{i n}-S(t)\right]-\rho_{m} \frac{S(t)}{S(t)+K_{S}} C(t) \\
\dot{N}(t)= & -D N(t)+\rho_{m} \frac{S(t)}{S(t)+K_{S}} C(t) \\
& -\gamma(I) N(t) \frac{L(t)}{C(t)}+\beta L(t) \\
\dot{L}(t)= & -D L(t)+\gamma(I) N(t) \frac{L(t)}{C(t)}-\beta L(t) \\
\dot{C}(t)= & -D C(t)+a(I) L(t)-\lambda C(t)
\end{aligned}\right.
$$

\section{QUALITATIVE PROPERTIES}

The analysis of the qualitative properties allows a structural validation of the model, independently from the parameter values. The aim of this analysis is to determine whether the steady inputoutput properties of the model are consistent with the observations.

\subsection{Determination of the equilibria}

In this step, we compare the steady-state model outputs and some of their ratios with their experimental equivalent for different dilution rates and light intensities. Relationships are expressed as functions of $I$ and $D$.

Model solutions at steady-state are marked with $\star$. The $L^{\star}: C^{\star}$ ratio is deduced from $\dot{C}(t)=0$ :

$$
\frac{L^{\star}}{C^{\star}}=\frac{D+\lambda}{a(I)}
$$

The $N^{\star}: C^{\star}$ ratio is calculated from the equation $\dot{L}(t)=0$ :

$$
\frac{N^{\star}}{C^{\star}}=\frac{D+\beta}{\gamma(I)}
$$

The steady-state solutions of $S^{\star}$ and $N^{\star}$ are obtained by combining the equations $\dot{N}(t)=0$ and $\dot{S}(t)=0$ :

$$
\begin{gathered}
S^{\star}=\frac{K_{S} D[k(I)(D+\lambda)+(D+\beta)]}{\rho_{m} \gamma(I)-D[k(I)(D+\lambda)+(D+\beta)]} \\
N^{\star}=\frac{(D+\beta)\left(S_{i n}-S^{\star}\right)}{k(I)(D+\lambda)+(D+\beta)}
\end{gathered}
$$

$C^{\star}$ is obtained from equations (6) and (8):

$$
C^{\star}=\frac{\gamma(I)\left(S_{i n}-S^{\star}\right)}{k(I)(D+\lambda)+(D+\beta)}
$$

$L^{\star}$ is finally deduced from equations (5) and (9):

$$
L^{\star}=\frac{k(I)(D+\lambda)\left(S_{i n}-S^{\star}\right)}{k(I)(D+\lambda)+(D+\beta)}
$$

\subsection{Qualitative steady input-output behaviour}

It is worth noting that measurements of $S^{\star}$ are always much smaller than $S_{i n}$. The value of $S^{\star}$ is linearly related to $K_{S}$ (see equation (6)) and $K_{S}$ does not intervene for the computation of the other equilibrium states. Therefore the predicted value of $S^{\star}$ can be tuned by $K_{S}$ : a small value of $K_{S}$ will ensure that $S^{\star}$ computed from the model is very low. We will therefore approximate $S_{\text {in }}-S^{\star} \simeq S_{i n}$. It follows that:

$$
N^{\star} \simeq \frac{(D+\beta) S_{i n}}{k(I)(D+\lambda)+(D+\beta)}
$$

If the pigment degradation rate is higher than the respiration rate $(\beta>\lambda)$, then $N^{\star}$ is a decreasing function with respect to light and dilution rate. We will make this assumption in the sequel.

We have then:

$$
C^{\star} \simeq \frac{\gamma(I) S_{i n}}{k(I)(D+\lambda)+(D+\beta)}
$$

and:

$$
L^{\star} \simeq \frac{k(I)(D+\lambda) S_{i n}}{k(I)(D+\lambda)+(D+\beta)}
$$

By differentiating the expression (5) with respect to light, it is straightforward to see that $\frac{N^{\star}}{C^{\star}}$ is minimal when $I_{o p t}=\sqrt{K_{I} \cdot K_{C}}$. For lower intensities, this ratio is decreasing when light increases. The ratio $\frac{N^{\star}}{C^{\star}}$ decreases with light and increases with dilution rate.

The qualitative behaviour of the state variables and of the considered ratios, when $I$ or $D$ increase, 
is summarized in Table 1 . Figure 1 represents the variations of particulate carbon and chlorophyll concentrations for different dilution rates. In conclusion the proposed model has the same observed steady input-output behaviour as observations in the photobioreactor.

\begin{tabular}{|c|c|c|}
\hline Quantity & $I$ & $D$ \\
\hline$C^{\star}$ & + & - \\
\hline$L^{\star}$ & - & + \\
\hline$N^{\star}$ & + & - \\
\hline$\frac{N^{\star}}{C^{\star}}$ & - & + \\
\hline$\frac{L^{\star}}{C^{\star}}$ & $-\&+$ & + \\
\hline
\end{tabular}

Table 1. Qualitative input-output behaviour of the model: evolution of the steady-state values with respect to $I$ and $D$ (+: increasing, :decreasing, $-\&+$ : decreasing then increasing).

\section{QUANTITATIVE PROPERTIES}

We propose here to use the steady input-output behaviour in order to identify the model parameters. We claim that this method provides better results than the classical global approach that consists in minimizing a criterion (e.g. least square between simulations and data) with respect to the parameter vector. Indeed, for biological systems whose models are rough approximations and whose measurements are noisy, the global minimization method leads most of the time to local minima. The proposed approach allows to decouple the parameter estimations (1.e. they are identified by small sets) and provides at the same time a way to validate the model. The approach is based on a quantitative analysis of the steady input-output behaviour. For sake of conciseness, we will explain the idea of the method with an example.

5.1 Use of the steady input output behaviour for identification

We will use the steady input-output behaviour obtained for the ratio $\frac{L^{\star}}{C^{\star}}$ given by equation (5) and rewritten here as a function of the inputs $I$ and $D$ :

$$
\frac{L^{\star}}{C^{\star}}=\frac{K_{I} \cdot D}{\alpha \cdot I}+\frac{D}{\alpha}+\frac{\lambda \cdot K_{I}}{\alpha \cdot I}+\frac{\lambda}{\alpha}
$$

This expression can be seen as a linear relationship between the steady ratio $\frac{L^{\star}}{C^{\star}}$, and the combinations of inputs given by $\frac{D}{I}, D$ and $\frac{1}{I}$. Therefore, there is a regression between these known quantities and the unknown ratios $\frac{K_{I}}{\alpha}, \frac{1}{\alpha} \frac{\lambda K_{I}}{\alpha}$ and $\frac{\lambda K_{I}}{\alpha}$. Theorically, a least square minimization of this regression for more than 4 independent data sets (i.e. measurements of the ratio $\frac{L^{\star}}{C^{\star}}$ ) obtained for various inputs will provide an estimate of $\frac{K_{I}}{\alpha}, \frac{1}{\alpha} \frac{\lambda K_{I}}{\alpha}$ and $\frac{\lambda K_{I}}{\alpha}$. From these ratios it is straightforward to obtain the values of $\alpha, \lambda$ and $K_{I}$. Nevertheless this method involves many unknowns in the regression, and therefore we propose to improve it as follows. We will consider equation (13) obtained for 2 different values of $I$, denoted $I_{1}$ and $I_{2}$. The difference between the values of $\frac{L^{\star}}{C^{\star}}$ obtained for the same dilution rate $D$ is given by:

$$
\frac{\left.\frac{L^{\star}}{C^{\star}}\right|_{I_{1}}-\left.\frac{L^{\star}}{C^{\star}}\right|_{I_{2}}}{\frac{1}{I_{1}}-\frac{1}{I_{2}}}=D \cdot \frac{K_{I}}{\alpha}+\frac{K_{I} \cdot \lambda}{\alpha}
$$

It is worth noting that the terms $\frac{D}{\alpha}$ and $\frac{\lambda}{\alpha}$ disappeared in equation (14). The new relation is expressed as a linear function of $D$. A simple linear regression using experimental data will then provide an estimate of $\lambda$. Of course, $\alpha$ and $K_{I}$ can not be directly evaluated from this regression, but from other relationships. In particular, the same approach can be used with the difference between ratios $\frac{L^{\star}}{C^{\star}}$ obtained for the same dilution rate and for various light intensities. The significance of the regression with respect to the data may be statistically checked to assess the quantitative validity of the steady input-output behaviour. Except $\rho_{m}$ and $K_{S}$, all parameters have been identified with this method. $\rho_{m}$ and $K_{S}$ are identified in batch conditions (dilution rate is zero) at the beginning of each experiments since the kinetic of nitrate absorption is adequately estimated only when the nitrate concentration is detectable (after it falls under the detection threshold).

Only after this step of steady-state identification, parameters tuning is improved by a global least square minimization, starting with initial parameter values provided by the previous approach. The resulting values of the parameters are represented in Table 2. They do not differ greatly from the values obtained by the exploitation of the steady input-output behaviour.

\begin{tabular}{|c|c|c|}
\hline parameters & values & units \\
\hline$\rho_{m}$ & 0.5 & $\mu$ molN.molC ${ }^{-1} . \mathrm{d}^{-1}$ \\
\hline$K_{S}$ & 0.43 & $\mu \mathrm{M} \mathrm{N}$ \\
\hline$K_{L}$ & 6.59 & n.d. \\
\hline$K_{C}$ & 33.0 & $\mu$ mol quanta.m ${ }^{-2} . \mathrm{s}^{-1}$ \\
\hline$\alpha$ & 24.1 & $\mathrm{~d}^{-1}$ \\
\hline$K_{I}$ & 208.5 & $\mu$ mol quanta.m \\
\hline$\lambda$ & 0.054 & $\mathrm{~d}^{-1}$ \\
\hline$\beta$ & 0.345 & $\mathrm{~d}^{-1}$ \\
\hline
\end{tabular}

Table 2. Estimates of the model parameters.

\subsection{Dynamic simulations}

Dynamic simulations are compared to experimental time series. The model is integrated with a second order Runge-Kutta method with variable 
step time. Three criteria are used to evaluate the model:

1) the difference between steady observations and model predictions.

2) the ability to describe transient phases obtained between two steady-states when the dilution rate has been modified.

3) the ability to describe batch mode results.

Chlorophyll and carbon predictions are close to experiments for steady-state conditions (see Fig. 2 ) but in some extreme cases of very low or high dilution or light intensities, the output of chlorophyll and carbon may be under- or overestimated. Transient phases are evolving accordingly to the experimental data. Carbon predictions are particularly close to measurements. For Chlorophyll, the model reproduces less precisely measurements.
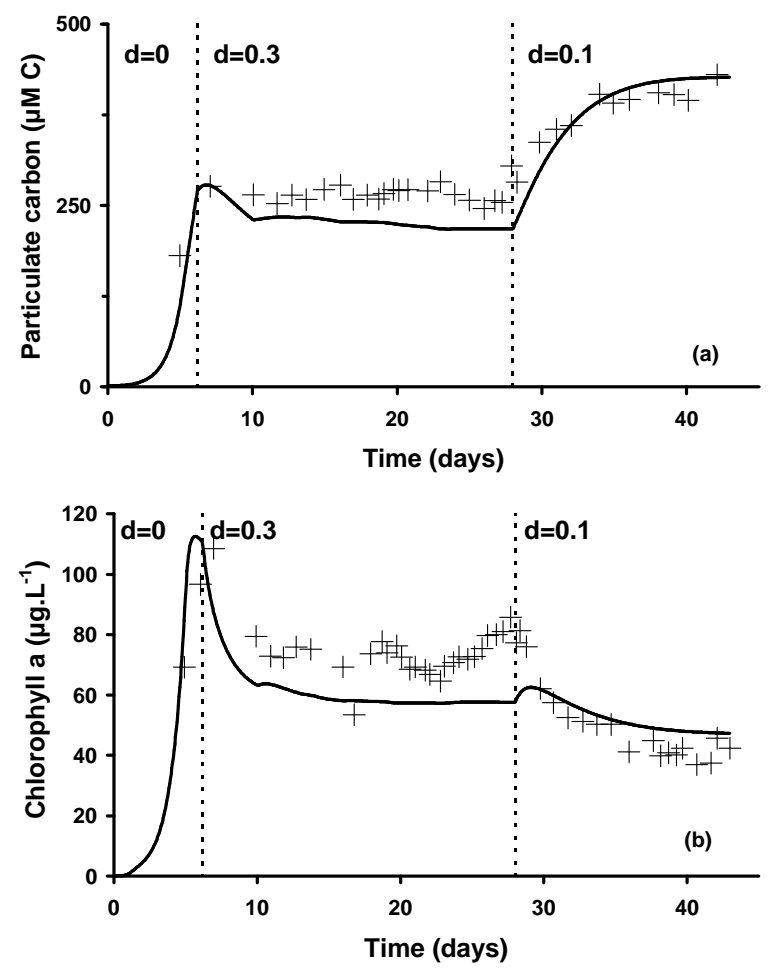

Fig. 2. Comparison of experimental data and model output for particular carbon (a) and chlorophyll (b). The light intensity of the experiment is $32 \mu \mathrm{mol}$ quanta. $\mathrm{m}^{-2} \cdot \mathrm{s}^{-1}$. The dilution rate is successively set to $0,0.3$ and $0.1 \mathrm{~d}^{-1}$.

\section{CONCLUSION}

Although this model is made of simple assumptions and has few parameters, it reproduces correctly the qualitative and quantitative inputoutput behaviour observed in the experiments. More generally, we have demonstrated in this approach the utility of the steady input-output behaviour in order to validate and calibrate a biological model.

Acknowledgement: We thank the Action "Bioinformatique" of the CNRS (SEMPO) and the Colors action (Hydrobio) for their fundings.

\section{REFERENCES}

Bastin, G. and D. Dochain (1990). On-line estimation and adaptive control of bioreactors. Elsevier.

Bernard, O., G. Malara and A. Sciandra (1996). The effects of a controlled fluctuating nutrient environment on continuous cultures of phytoplankton monitored by a computer. J. Exp. Mar. Biol. Ecol. 197, 263-278.

Chalup, M.S. and E.A. Laws (1990). A test of the assumptions and predictions of recent microalgal growth models with the marine phytoplankter Pavlova lutheri. Limnol. Oceanogr. 35(3), 583-596.

Dugdale, R. C. (1967). Nutrient limitation in the sea: dynamics, identification and significance. Limnol. Oceanogr. 12, 685-695.

Falkowski, P.G. and T.G. Owens (1980). Lightshade adaptation : two strategies in marine phytoplankton. Plant Physiol. 66, 592-595.

Geider, R.J., H.L. Macintyre and T.M. Kana (1998). A dynamic regulatory model of phytoplanktonic acclimatation to light, nutrients and temperature. Limnol. Oceanogr. 43(4), 679-694.

Laws, E.A. and T.T. Bannister (1980). Nutrientand light-limited growth of Thalassiosira fluviatilis in continuous culture with implications for phytoplankton growth in the ocean. Limnol. Oceanogr. 25(3), 457-473.

Rhee, G.Y. and I.J. Gotham (1981). The effect of environmental factors on phytoplankton growth: light and the interactions of light with nitrate limitation. Limnol. Oceanogr. 26(4), 649-659.

Sciandra, A., J. Gostan, Y. Collos, C. DescolasGros, C. Leboulanger, V. Martin-Jezequel, M. Denis, D. Lefevre, C. Copin-Montegut and B. Avril (1997). Growth-compensating phenomena in continuous cultures of Dunaliella tertiolecta limited simultaneously by light and nitrate. Limnol. oceanogr. 42(6), 13251339 .

Sciandra, A., L. Lazzara, H. Claustre and M. Babin (2000). Responses of the growth rate, pigment composition and optical properties of Cryptomonas sp. to light and nitrogen stresses. Mar. Ecol. Prog. Ser. 201, 107120 .

Shuter, B. (1979). A model of physiological adaptation in unicellular algae. J. Theor. Biol. $\mathbf{7 8}, 519-552$. 